\title{
Manejo de pacientes con trastornos mentales en servicios ambulatorios de Medicina General en tres hospitales de Lima.
}

Care of patients with mental disorders in general medicals outpatient settings in three hospitals in Lima.

DE LA GRECCA PREVOO Robert ${ }^{1}$, ZAPATA VEGA María Isabel ${ }^{2}$, VEGA DIENSTMAIER Johann ${ }^{3}$, MAZZOTTI Guido $\uparrow$.

\section{SUMMARY}

The international literature reports different studies on the management of psychiatric disorders (PD) in non-psychiatric settings; however, in Peru the studies are basically focused on the prevalence of PD in general population or in non-psychiatric hospital settings. Objective: To collect information about characteristics and attitudes of General Practitioners (GPs) and patterns in managing patients with possible psychiatric disorders (PD). Material and methods: A survey was administered to 73 physicians working in general medical outpatient settings in three hospitals in Lima-Peru. Results: $23 \%$ of GPs received postgraduate psychiatry update courses, $56 \%$ felt confident in their ability to diagnose a patient with a PD, $82 \%$ reported interest in their medical management, and $66 \%$ felt capable of helping them. As their usual practice, $37 \%$ reported performing a mental status examination (MSE), $48 \%$ asking about sexual issues, and $37 \%$ about personal and family history of PD. Confidence in their diagnosis was significantly higher in male GPs and attendings, but interest in the management was higher in females. Post-graduate psychiatric update was significantly associated with confidence in diagnosing PD, asking about psychiatric history and sexual health, and performing MSE. Most frequently seen PD listed were: depression, unexplained somatic complaints, and anxiety disorders. Most frequently prescribed psychotropics listed were: hypnotics/sedatives; tonics, vitamins, and herbs; and antidepressants, particularly tricyclics. If a diagnosis of PD was made, 93\% referred the patients to a psychiatrist. Conclusions: Factors related to the GPs may influence the patterns of care of psychiatric patients in primary care. (Rev Med Hered 2005;16:246-252).

KEYWORDS: Mental disorders, health services, primary health care.

\section{RESUMEN}

La literatura mundial describe diversos estudios sobre el manejo de trastornos mentales (TM) en servicios no psiquiátricos; sin embargo, en el Perú los estudios están básicamente dirigidos a determinar la prevalencia de trastornos mentales en población general o en población hospitalaria no-psiquiátrica. Objetivo: Obtener información sobre las características y actitudes de los médicos generales (MGs) y los patrones de manejo de pacientes con probables TM. Materiales y métodos: Se administró una encuesta a 73 médicos que trabajaban en servicios ambulatorios de medicina general en tres hospitales de Lima-Perú. Resultados: El 23\% de los MGs recibieron algún curso de salud mental luego de haber concluido los estudios universitarios, 56\% se siente confiado en su propia habilidad para diagnosticar un TM, 82\% reporta interés en manejar médicamente a pacientes con TM y $66 \%$ de siente capaz de

\footnotetext{
Médico Residente de Administración de Salud, Universidad Peruana Cayetano Heredia, Lima, Perú. Profesor Asistente, Departamento de Psiquiatría, Mount Sinai School of Medicine, New York, USA. Profesor del Departamento de Psiquiatría y Salud Mental, Universidad Peruana Cayetano Heredia, Lima, Perú.

$\dagger \quad$ In Memoriam.

246 Rev Med Hered 16 (4), 2005
} 
ayudarlos. Como práctica usual, 37\% reporta realizar evaluación del estado mental (EEM), 48\% dice preguntar sobre conducta sexual y $37 \%$ sobre historia personal y familiar de TM. La confianza en hacer un diagnóstico fue significativamente más elevada en MGs varones y en asistentes, pero el interés para manejarlos médicamente fue mayor en MGs mujeres. La actualización en salud mental en post-grado se asoció significativamente con la confianza en diagnosticar TM, preguntar sobre historia psiquiátrica y salud sexual, y realizar EEM. Los TM listados como más frecuentemente vistos fueron: depresión, trastornos somáticos sin explicación y trastornos de ansiedad. Los psicotrópicos listados como más utilizados fueron: hipnóticos/sedantes; tónicos, vitaminas y hierbas; y antidepresivos, particularmente tricíclicos. Diagnosticado un TM, 93\% dice referirlo a un psiquiatra. Conclusiones: Factores relacionados a los MGs pueden influenciar los patrones de tratamiento de pacientes psiquiátricos en atención primaria. (Rev Med Hered 2005;16:246-252).

PALABRAS CLAVE: Trastornos mentales, servicios de salud, atención primaria de salud.

\section{INTRODUCCIÓN}

Aproximadamente una cuarta parte de pacientes que acuden a servicios de atención primaria sufren algún trastorno mental. Esto significa que una proporción importante de personas con trastornos mentales es vista inicialmente por médicos no-psiquiatras y la mayoría de ellos (69\% a nivel mundial), se presentarían con síntomas somáticos como queja principal, dificultando su detección y adecuada atención (1).

La literatura mundial describe como causas probables de la falla en el diagnóstico de trastornos mentales: la presencia de sintomatología somática, actitudes del médico hacia los pacientes y sus problemas mentales, el manejo de la entrevista psiquiátrica $(2,3,4,5)$ y las características de la personalidad del médico $(6,7)$. Por otro lado, se ha demostrado que el haber llevado entrenamiento en tópicos de psiquiatría mejora la habilidad del médico general para la detección de casos de trastornos mentales (8). Se ha sugerido que las causas de estos problemas estarían relacionadas a fallas en la educación médica (a nivel de estudiantes y residentes) sobre el reconocimiento y tratamiento adecuado de los trastornos mentales (9).

Si bien en la literatura mundial el manejo de trastornos mentales en servicios no-psiquiátricos ha sido ampliamente estudiado; en el Perú, los estudios están básicamente dirigidos a determinar la prevalencia de trastornos mentales en población general $(10,11,12$, $13,14,15)$ o en población hospitalaria no-psiquiátrica, particularmente orientados a la prevalencia de depresión $(16,17,18)$. El objetivo del presente estudio fue obtener información sobre las características y actitudes de los médicos generales (MGs) y los patrones de manejo de pacientes con probables trastornos mentales en servicios ambulatorios de medicina general. Entendemos como patrones de manejo a toda intervención terapéutica incluyendo la evaluación diagnóstica y tratamiento del posible trastorno.

\section{MATERIALES Y MÉTODOS}

Se administró una encuesta a los médicos asistentes y residentes de los programas de medicina interna en el Hospital Arzobispo Loayza (HAL), Hospital Nacional Cayetano Heredia (HNCH) y Hospital General María Auxiliadora (HGMA) en abril y mayo de 1998. Se realizó el estudio en estos 3 hospitales debido a que dieron las facilidades y permisos necesarios para la ejecución del trabajo. Se incluyó a la totalidad de médicos de cada hospital que realizaban consulta ambulatoria regular de medicina interna y/o general, los cuales expresaron su consentimiento verbal para participar en el estudio.

El instrumento utilizado en este estudio, fue un cuestionario de 28 preguntas (19) que indagan datos del médico, su actitud e interés por los pacientes con trastornos mentales, las características de su atención ambulatoria, el manejo en general que se da a los pacientes que acuden a consulta, los diagnósticos psiquiátricos que realiza en un mes promedio y el manejo que da a los pacientes psiquiátricos en general. Este cuestionario fue modificado sobre el utilizado como antecedente para la elaboración del borrador de la Versión de la CIE - 10 para la Atención Primaria de Salud de la OMS.

Las encuestas fueron entregadas personalmente a cada médico acompañándose de una carta de presentación donde se daban las instrucciones. Luego de completar las encuestas, éstas fueron recogidas en un sobre cerrado por los encuestadores para asegurar la confidencialidad del médico. 
El análisis estadístico de datos se realizó con el programa SPSS versión 12.

\section{RESULTADOS}

La muestra constó de 73 médicos, 54,8\% del HAL $(\mathrm{n}=40), 35,6 \%$ del HNCH $(\mathrm{n}=26)$ y $9,6 \%$ del HGMA $(\mathrm{n}=7)$. Los médicos asistentes representaron el $41 \%$ $(\mathrm{n}=30)$ y los médicos residentes el 59\% $(\mathrm{n}=43)$. La gran mayoría de médicos participantes, pertenecían al sexo masculino $(76,7 \%, \mathrm{n}=56)$. La edad promedio de la muestra fue de 36,3 $\pm 7,63$ años (asistentes: 42,6 $\pm 7,58$ años; residentes: $31,6 \pm 2,62$ años). El tiempo promedio de graduado fue de 9,2 $\pm 7,43$ años) (asistentes: $16 \pm 7,17$ años ; residentes: 4,49 $\pm 1,82$ años).

Solamente el $23,3 \%$ de los médicos $(n=17)$ reportó haber recibido algún curso de actualización en psiquiatría o salud mental desde que concluyó sus estudios en la escuela de medicina; lo que a su vez representó el 36,7\% de los médicos asistentes y el 14\% de los médicos residentes con una diferencia significativa $(\mathrm{p}=0,024)\left(\right.$ Tabla $\left.\mathrm{N}^{\circ} 1\right)$.

La confianza en hacer un diagnóstico estuvo significativamente asociada a los médicos de sexo masculino $(\mathrm{p}=0,048)$ y a los médicos asistentes $(\mathrm{p}=$ $0,003)$, pero el interés para manejarlos médicamente fue mayor en médicos de sexo femenino $(\mathrm{p}=0,03)$ (Tabla $\mathrm{N}^{\circ} 1$ ).

Recibir cursos de actualización en salud mental en el post-grado se asoció significativamente con la confianza en la propia habilidad para diagnosticar trastornos mentales $(\mathrm{p}=0,013)$. Adicionalmente, se observó que la actualización en salud mental se asocia a patrones de obtención de datos clínicos que permitirían la detección de posibles casos de trastornos mentales, como preguntar sobre historia personal y familiar de enfermedad mental ( $p<0,001)$, indagar sobre conducta sexual $(p=0,007)$ y realizar evaluación del estado mental de los pacientes $(\mathrm{p}=0,001)\left(\right.$ Tabla $\left.\mathrm{N}^{\circ} 2\right)$.

El $84,9 \%$ del total de médicos consideró que no contaba con el tiempo suficiente para interrogar y

Tabla $\mathbf{N}^{\circ} 1$. Factores relacionados al médico frente a los pacientes con trastornos mentales.

\begin{tabular}{|c|c|c|c|c|c|}
\hline & $\begin{array}{l}\text { Total de } \\
\text { Médicos } \\
\mathbf{n}=73 \\
\%(n) \\
\end{array}$ & $\begin{array}{c}\text { Médicos } \\
\text { Asistentes } \\
\text { n=30 } \\
\% \text { (n) }\end{array}$ & $\begin{array}{c}\text { Médicos } \\
\text { Residentes } \\
\text { n=43 } \\
\%(n)\end{array}$ & $\begin{array}{l}\text { Médicos } \\
\text { Varones } \\
\mathbf{n = 5 6} \\
\%(\mathbf{n}) \\
\end{array}$ & $\begin{array}{c}\text { Médicos } \\
\text { Mujeres } \\
\mathbf{n}=17 \\
\%(\mathbf{n})\end{array}$ \\
\hline $\begin{array}{l}\text { Recibió curso de } \\
\text { actualización en tópicos } \\
\text { de psiquiatría en el } \\
\text { post-grado }\end{array}$ & $23,3 \%(17)$ & $36,7 \%(11)^{\mathrm{A}}$ & $14,0 \%(6)^{\mathrm{A}}$ & $23,2 \%(13)$ & $23,5 \%$ (4) \\
\hline $\begin{array}{l}\text { Confía en su propia } \\
\text { habilidad para hacer un } \\
\text { diagnóstico de trastorno } \\
\text { mental }\end{array}$ & $56,2 \%(41)$ & $76,7 \%(23)^{\mathrm{B}}$ & $41,9 \%(18)^{\mathrm{B}}$ & $62,5 \%(35)^{\mathrm{C}}$ & $35,3 \%(6)^{\mathrm{C}}$ \\
\hline $\begin{array}{l}\text { Tiene interés por los } \\
\text { pacientes con trastornos } \\
\text { mentales }\end{array}$ & $82,2 \%(60)$ & $86,7 \%(26)$ & $79,1 \%(34)$ & $76,8 \%(43)^{\mathrm{D}}$ & $100 \%(17)^{\mathrm{D}}$ \\
\hline $\begin{array}{l}\text { Cree que los pacientes } \\
\text { con trastornos mentales } \\
\text { pueden ser ayudados }\end{array}$ & $65,8 \%(48)$ & $76,7 \%(23)$ & $58,1 \%(25)$ & $64,3 \%(36)$ & $70,6 \%(12)$ \\
\hline $\begin{array}{l}\text { Indaga sobre } \\
\text { antecedentes de } \\
\text { trastornos mentales }\end{array}$ & $37,0 \%(27)$ & $46,7 \%(14)$ & $30,2 \%(13)$ & $37,5 \%(21)$ & $35,3 \%(6)$ \\
\hline $\begin{array}{l}\text { Indaga sobre conducta } \\
\text { sexual }\end{array}$ & $47,9 \%(35)$ & $43,3 \%(13)$ & $51,2 \%(22)$ & $46,4 \%(24)$ & $52,9 \%(9)$ \\
\hline $\begin{array}{l}\text { Realiza evaluación del } \\
\text { estado mental }\end{array}$ & $37 \%(27)$ & $50,0 \%(15)$ & $27,9 \%(12)$ & $32,1 \%(18)$ & $52,9 \%(9)$ \\
\hline $\begin{array}{l}=0,024(\text { Chi-cuadrado }) \\
=0,048(\text { Chi-cuadrado }) \\
D\end{array}$ & $\begin{array}{l}0,003 \text { (Chi-cu } \\
\text {,03 (Prueba }\end{array}$ & de Fisher) & & & \\
\hline
\end{tabular}


Tabla $\mathbf{N}^{\circ} 2$. Factores relacionados con recibir cursos de actualización en salud mental en el post-grado.

\begin{tabular}{|c|c|c|c|}
\hline $\begin{array}{c}\text { Recibieron cursos de actualización en } \\
\text { salud mental }\end{array}$ & $\begin{array}{c}\text { No } \\
(\mathbf{n}=56) \\
\% \quad(n)\end{array}$ & $\begin{array}{c}\text { Sí } \\
(\mathrm{n}=17) \\
\% \quad(\mathbf{n})\end{array}$ & $\mathbf{p}$ \\
\hline $\begin{array}{l}\text { Confía en su propia habilidad para hacer un } \\
\text { diagnóstico de trastorno mental }\end{array}$ & $48,2 \%(27)$ & $82,4 \%(14)$ & $0,013^{\mathrm{A}}$ \\
\hline $\begin{array}{l}\text { Tiene interés por los pacientes con } \\
\text { trastornos mentales }\end{array}$ & $78,6 \%(44)$ & $94,1 \%(16)$ & $0,276^{\mathrm{B}}$ \\
\hline $\begin{array}{l}\text { Cree que los pacientes con trastornos } \\
\text { mentales pueden ser ayudados }\end{array}$ & $64,3 \%(36)$ & $70,6 \%(12)$ & $0,631^{\mathrm{A}}$ \\
\hline $\begin{array}{l}\text { Indaga sobre antecedentes de trastornos } \\
\text { mentales }\end{array}$ & $25,0 \%(14)$ & $76,5 \%(13)$ & $<0,001^{\mathrm{A}}$ \\
\hline Indaga sobre conducta sexual & $39,3 \%(22)$ & $76,5 \%(13)$ & $0,007^{\mathrm{A}}$ \\
\hline Realiza evaluación del estado mental & $25,0 \%(14)$ & $76,5 \%(13)$ & $0,001^{\mathrm{A}}$ \\
\hline
\end{tabular}

A (Chi-Cuadrado) B (Prueba Exacta de Fisher)

examinar a sus pacientes.

El $42,3 \%$ de los médicos refirieron haber citado alguna vez a pacientes exclusivamente para sesiones de orientación o consejería, siendo la frecuencia promedio de citas de $1.01 \pm 1,90$ veces por mes. El $40,8 \%$ de los médicos refieren pedir al paciente traer al cónyuge u otro familiar para ayudar a solucionar algún problema psicológico, siendo la frecuencia promedio de $0,87 \pm 1,51$ sesiones por mes. Se encontró que aquellos médicos que habían recibido cursos de actualización en el postgrado citan con mayor frecuencia a los familiares en un promedio de 2,06 $\pm 2,46$ pacientes por mes, mientras que los que no recibieron cursos de actualización reportaron citar a la familia con un promedio de $0,53 \pm 0,86$ pacientes por mes ( $\mathrm{p}<0,026$, Prueba T de Student).

Al indagar sobre los trastornos mentales más frecuentemente vistos en la consulta ambulatoria, los médicos participantes citaron depresión, quejas somáticas sin explicación y trastornos de ansiedad como los más frecuentes (Tabla $\mathrm{N}^{\circ} 3$ ).

Con respecto a los psicofármacos más recetados, los médicos participantes listaron con mayor frecuencia el uso de fármacos sedantes e hipnóticos; otras sustancias como tónicos, vitaminas y hierbas; y antidepresivos. Entre los antidepresivos, el uso de tricíclicos se registró con mayor frecuencia que los "nuevos" antidepresivos, tales como la fluoxetina (Tabla $\left.\mathrm{N}^{\circ} 4\right)$.

Ante un diagnóstico o una fuerte sospecha de trastorno mental, los encuestados procederían con la siguiente derivación: $91,3 \%$ al psiquiatra, 3,5\% al psicólogo, 1,3\% al neurólogo y un 3,5\% lo trataría el mismo médico.

\section{DISCUSIÓN}

Los principales hallazgos de este estudio pueden resumirse en que la condición de ser médicos varones, asistentes, y con cursos de actualización luego de terminada la carrera, se asocia significativamente a mayor confianza en los diagnósticos de algún trastorno mental y otros parámetros asociados a estos procedimientos. Por otro lado, ser médico mujer se asocia con un interés mayor en la atención de tales pacientes.

Consideramos que las principales limitaciones del presente trabajo son que el estudio no fue una observación directa de la evidencia, sino que recogió datos referidos por los médicos. Adicionalmente, esta información fue recolectada en 1998 por lo que se recomendaría un seguimiento en diferentes períodos de tiempo, en poblaciones similares, para evidenciar posibles cambios en las intervenciones médicas con la disponibilidad y utilización de nuevas modalidades terapéuticas.

El hallazgo de que haber seguido cursos de actualización en psiquiatría o salud mental luego de finalizar los estudios universitarios, se asocia con la confianza y capacidad para realizar el diagnóstico, con mejor obtención de datos importantes y con el hecho de involucrar a la familia del paciente en el diagnóstico y tratamiento del trastorno mental, es consistente con 
Tabla $N^{\circ}$ 3. Pacientes con posibles trastornos mentales reportados por los médicos del estudio.

\begin{tabular}{lccc}
\hline \multicolumn{1}{c}{ Trastornos mentales } & $\begin{array}{c}\text { Porcentaje } \\
\text { promedio } \\
\text { pacientes con } \\
\text { posibles } \\
\text { trastornos } \\
\text { mentales }\end{array}$ & $\begin{array}{c}\text { Frecuencia } \\
\text { mensual de } \\
\text { pacientes } \\
\text { diagnosticados } \\
\text { con trastornos } \\
\text { mentales (DE) }\end{array}$ & $\begin{array}{c}\text { Médicos que } \\
\text { dicen hacer el } \\
\text { diagnóstico de } \\
\text { trastornos } \\
\text { mentales( } * *)\end{array}$ \\
& $($ DE)(*) & & \\
Depresión & & & \\
Quejas somáticas serias sin explicación adecuada & $10,95(13,44)$ & $7,53(7,38)$ & $95,9 \%$ \\
Trastorno de ansiedad & $10,64(14,76)$ & $7,69(9,67)$ & $83,3 \%$ \\
Trastorno del sueño & $8,61(12,27)$ & $6,11(7,46)$ & $84,7 \%$ \\
Trastornos disociativos/conversivos & $6,96(8,47)$ & $5,33(6,73)$ & $80,6 \%$ \\
Trastornos de personalidad & $4,48(9,60)$ & $2,99(4,87)$ & $64,4 \%$ \\
Alcoholismo y uso de drogas & $3,16(7,27)$ & $1,89(3,30)$ & $50,7 \%$ \\
Trastornos de la conducta alimenticia & $2,48(3,46)$ & $1,72(2,16)$ & $61,1 \%$ \\
Trastornos sexuales & $2,41(5,60)$ & $1,85(4,54)$ & $40,3 \%$ \\
Delirio y Demencia & $1,97(6,37)$ & $1,27(2,95)$ & $34,2 \%$ \\
Trastorno bipolar & $1,31(2,48)$ & $1,12(2,10)$ & $38,4 \%$ \\
Trastornos psicóticos & $0,86(2,00)$ & $0,58(1,34)$ & $24,7 \%$ \\
& $0,71(1,79)$ & $0,51(1,09)$ & $29,2 \%$
\end{tabular}

(*)Frecuencia mensual de pacientes con posibles trastornos mentales vistos por los médicos generales dividido entre el numero total de pacientes vistos por mes.

(**) Por lo menos una vez al mes.

Tabla $\mathbf{N}^{\circ}$ 4. Psicotrópicos prescritos reportados por los médicos del estudio.

\begin{tabular}{lcc}
\hline \multicolumn{1}{c}{ Psicotrópicos } & $\begin{array}{c}\text { Psicotrópicos } \\
\text { promedio / mes } \\
(\mathbf{D E})(*)\end{array}$ & $\begin{array}{c}\text { \% de médicos } \\
\text { que recetan el } \\
\text { fármaco }(* *)\end{array}$ \\
\hline & $11,44(10,77)$ & \\
Benzodiazepinas (Sedantes/Hipnóticos) & $5,00(9,19)$ & 91,8 \\
Tónicos, vitaminas y hierbas & $3,13(4,11)$ & 54,2 \\
Antidepresivos tricíclicos & $2,41(3,39)$ & 60,6 \\
Nuevos antidepresivos (Fluoxetina, etc.) & $0,51(2,46)$ & 64,8 \\
Beta-bloqueadores (para ansiedad) & $0,28(1,24)$ & 14,1 \\
Neurolépticos orales & $0,13(0,44)$ & 12,5 \\
Opioides (morfina) & $0,01(0,12)$ & 1,4 \\
Litio & 0 & 0,0 \\
Otros: neurolépticos de depósito, estimulantes & & \\
(anfetaminas, etc.) & & \\
(*) Frecuencia mensual promedio de fármacos que los médicos refieren prescribir. & \\
(**) Porcentaje del total de médicos que refieren indicar dichos fármacos psicotrópicos al menos 1 vez al mes.
\end{tabular}

lo descrito por Joukamaa et al, quienes encuentran que el haber llevado entrenamiento adicional en psiquiatría durante el post-grado permite una mayor habilidad para la detección de trastornos mentales (8). Del mismo modo, haber hecho un curso de actualización posterior a la culminación de los estudios universitarios implica la mejora en la técnica de entrevista, y en la obtención de mejores datos; Goldberg et al, encuentra que la obtención de datos (técnica de entrevista) es determinante en la habilidad del médico para detectar trastornos mentales y que ésta puede ser mejorada luego de un entrenamiento en tópicos específicos de entrevista psiquiátrica (20).

Se ha sugerido que gran parte del problema en la falla para la detección de trastornos mentales se relaciona con la actitud del médico frente a pacientes con estos trastornos; lo anterior influiría no sólo en el diagnóstico sino también en el tratamiento que el médico pueda prescribir (9). Se ha descrito que el comportamiento del médico frente al paciente influye en tal proceso; así, el estilo de entrevista y la interacción verbal con el 
paciente adquieren gran importancia y son resultado de la actitud que el médico pueda tener frente a los pacientes con trastornos mentales (5).

Los trastornos mentales referidos por los médicos del estudio como más frecuentes en la consulta ambulatoria fueron, en orden decreciente: depresión, las quejas somáticas serias sin explicación adecuada, trastornos de ansiedad, trastornos del sueño y trastornos disociativo/conversivos. Un estudio realizado en dos hospitales generales de Kuwait, documentó los casos de trastornos mentales registrados por 130 médicos internistas, encontrándose como trastornos mentales más frecuentes a los psicosomáticos, seguidos de depresión y ansiedad (21). Castillo y Alarcón, utilizando el SCL-90 en un estudio con pacientes de consulta ambulatoria del Hospital Nacional Cayetano Heredia de Lima; encuentran las siguientes patologías manifiestas: somatización, obsesión / compulsión, hostilidad y depresión; cabe hacer la diferencia que mientras en nuestro estudio así como en el estudio de Kuwait, los trastornos fueron referidos por los médicos que hacían la consulta ambulatoria, en el estudio de Castillo y Alarcón se aplicó un instrumento diagnóstico (SCL90) para determinar la patología psiquiátrica en la consulta ambulatoria y sin correlacionarse con la opinión o diagnóstico del médico tratante (17).

La mayoría de los médicos $(91,3 \%)$ del estudio refieren derivar los pacientes a un psiquiatra ante el diagnóstico o fuerte sospecha de trastorno mental. El estudio en Kuwait anteriormente mencionado refiere que más de un tercio de los pacientes diagnosticados de algún trastorno mental por los médicos internistas son tratados por ellos mismos (33\% de los trastornos ansiosos, $35 \%$ de los desórdenes psicosomáticos y $32 \%$ de los trastornos depresivos) disminuyendo de manera significativa el número de interconsultas a la especialidad de psiquiatría (21).

Es de notar que algo más de la mitad de médicos refiere utilizar medicamentos no psicotrópicos como tónicos, vitaminas y hierbas. Esto podría estar relacionado con la importante proporción de pacientes con quejas somáticas sin explicación médica adecuada, a quienes se indicaría un tratamiento (teóricamente) no nocivo. Otros psicotrópicos reportados como frecuentemente utilizados fueron las benzodiazepinas (utilizadas como hipnóticos o sedantes) y antidepresivos tricíclicos. Con relación al uso de benzodiazepinas, Jenks encuentra que en una proporción importante de atenciones los médicos generales prescribieron drogas psicotrópicas sin tener un diagnóstico de trastorno mental, principalmente benzodiazepinas. La explicación dada a este hallazgo fue que existían otras condiciones médicas donde pueden utilizarse estas drogas sin que necesariamente exista un trastorno mental (22).

Los hallazgos del presente estudio son un punto de partida para otros trabajos que investiguen sobre los patrones de atención de pacientes con probable patología psiquiátrica en servicios ambulatorios de medicina interna/general y a nivel de atención primaria en el sistema de salud en el Perú y Latinoamérica.

En conclusión, ciertas características de los médicos generales tales como el sexo, ser asistente y haber tenido cursos de actualización en psiquiatría, podrían influir en el manejo de pacientes con trastornos mentales en medicina general. La mayoría de los médicos del estudio refieren no tener tiempo adecuado para interrogar y examinar a sus pacientes. Los trastornos mentales más frecuentes en la consulta ambulatoria según los médicos del estudio son: depresión, quejas somáticas serias sin explicación, trastornos ansiosos y trastornos de sueño. Los medicamentos o productos más utilizados según los médicos del estudio son: sedantes/hipnóticos; tónicos, vitaminas y hierbas; y antidepresivos, particularmente los tricíclicos. La gran mayoría de los médicos del estudio prefiere derivar al psiquiatra a aquellos pacientes de quienes sospeche o haya hecho el diagnóstico de trastorno mental.

\section{Correspondencia:}

Dr. Robert De La Grecca Prevoo

Calle los Pinos D-10 La Molina

Lima 12 Perú

Teléfono: 99621998

Correo electrónico: robertodlg@yahoo.com

\section{REFERENCIAS BIBLIOGRÁFICAS}

1. WHO: The WHO education package on mental disorders in primary health care. WHO/MSA/MNH/ EAC/98.1. Geneva, 1998.

2. Goldberg D, Blackwell B. Psychiatric illness in general practice. BMJ 1970; 2: 439-443.

3. Harding T, De Aragón J, Baltazar J, et al. Mental disorders in primary care health: a study of their frequency and diagnosis in four developing countries. Psychol Med 1980; 10: 231-241. 
4. Jones R, Badger L, Ficken R, Leeper J, Anderson R. Inside the hidden mental health network. Gen Hosp Psychiatry 1987; 9: 287-293.

5. Schulberg H. Mental disorders in the primary care setting: Research priorities for de 1990's. Gen Hosp Psychiatry 1991; 13: 156-164.

6. Goldberg D, Steele J, Smith C, Spivey L. Training family doctors to recognize psychiatric illness with increased accuracy. Lancet 1980; 2: 521-523.

7. Goldberg D, Steele J, Johnson A, Smith C. Ability of primary care physicians to make accurate ratings of psychiatric symptoms. Arch Gen Psychiatry 1982; 39: 829-833.

8. Joukamaa M, Lehtinen V, Karlsson H. The ability of general practitioners to detect mental disorders in primary health care. Acta Psychiatr Scand 1995; 91: 52-56.

9. Lipowski Z. Is the education of primary care physicians adequate? Gen Hosp Psychiatry 1992; 14: 361-362.

10. Rotondo H. Morbilidad psiquiátrica en un área urbana en estado de desorganización. Revista Psiquiátrica Peruana 1959; 2: 132-137.

11. Rotondo H, Mariátegui J, Bambarén C. Estudios de morbilidad psiquiátrica en la población urbana de Mendocita. Revista Psiquiátrica Peruana 1959; 2: 242274.

12. Caravedo B, Valdivia O. Un estudio de salud mental en una muestra de población industrial. Revista Psiquiátrica Peruana 1960; 3: 47-64.

13. Mariátegui J, Alva V, De León O. Epidemiología psiquiátrica de un distrito urbano de Lima. Ediciones de la Revista de Neuropsiquiatría, 1969.
14. Hayashi S, Perales A, Sogi C, Warthon D, Llanos R. Prevalencia de vida de trastornos mentales en Independencia (Lima, Perú). Anales de Salud Mental 1985; 1: 206-222.

15. Perales A, Sogi C. Epidemiología psiquiátrica en el Perú. Anales de Salud Mental 1995; 11: 9-29.

16. Figueroa M, Pamo O. Prevalencia de síntomas depresivos en pacientes ambulatorios. Rev Med Hered 1991;1:31-34.

17. Castillo A, Alarcón R. Dimensiones psicopatológicas en pacientes no psiquiátricos. Revista de Neuropsiquiatría 1976; 39: 24-38.

18. Kufoy W. Determinación de niveles de depresión en pacientes con molestias gastrointestinales que acuden a consultorio de gastroenterología usando el BDI. Tesis de Bachiller en Medicina. Lima, Perú: Universidad Peruana Cayetano Heredia, 1984.

19. De la Grecca R. Características de la Atención Médica de los Trastornos Mentales en la Consulta Ambulatoria de Medicina Interna con Énfasis en Depresión en Tres Hospitales de Lima. Tesis para optar el titulo de Medico Cirujano, Lima, Perú, Universidad Peruana Cayetano Heredia, 1998.

20. Goldberg D, Steele J, Smith C. Teaching psychiatric interview techniques to family doctors. Acta Psychiatr Scand 1980; 62 (Suppl 285): 41-47.

21. Al-Ansari E, Khadadah M, Hassan K, Mirza I. Psychiatric disorders in two general hospitals. Gen Hosp Psychiatry 1991; 13: 319-324.

22. Jencks S. Recognition of mental distress and diagnosis of mental disorder in primary care. JAMA 1985; 253 : 1903-1907. 